\title{
Designing a Secure and Robust Mobile Interacting Robot for the Long Term
}

\author{
N. Tomatis ${ }^{\dagger \ddagger}$, G. Terrien ${ }^{\dagger}$, R. Piguet ${ }^{\dagger}$, D. Burnier ${ }^{\ddagger}$, S. Bouabdallah $^{\ddagger}$, Kai O. Arras ${ }^{\ddagger}$, R. Siegwart ${ }^{\ddagger}$ \\ ${ }^{\dagger}$ BlueBotics SA \\ PSE-C \\ CH-1015 Lausanne \\ n.tomatis@ieee.org \\ ${ }^{\ddagger}$ Autonomous Systems Lab, EPFL \\ Swiss Federal Institute of Technology Lausanne \\ $\mathrm{CH}-1015$ Lausanne \\ r.siegwart@ieee.org
}

\begin{abstract}
This paper presents the genesis of RoboX. This tour guide robot has been built from the scratch based on the experience of the Autonomous Systems Lab. The production of 11 of those machines has been realized by a spin-off of the lab: BlueBotics SA. The goal was to maximize the autonomy and interactivity of the mobile platform while ensuring high robustness, security and performance. The result is an interactive moving machine which can operate in human environments and interacts by seeing humans, talking to and looking at them, showing icons and asking them to answer its questions. The complete design of mechanics, electronics and software is presented in the first part. Then, as extraordinary test bed, the Robotics exhibition at Expo.02 (Swiss National Exhibition) permits to establish meaningful statistics over 5 months (from May 15 to October 20, 2002) with up to 11 robots operating at the same time.
\end{abstract}

\section{Introduction}

The task of a tour guide robot is to be able to move around autonomously in the environment, to acquire the attention of the visitors and to interact with them efficiently in order to fulfill its main goal: give the visitors a pre-defined tour. The environment is known and accessible, but a general approach requiring no environmental changes is better suited for a commercial purpose. For the same reason a fully-autonomous and self-contained robot is preferable. Furthermore such a machine is required to have a long live cycle and a high mean time between failure (MTBF), which minimizes the need of human supervision and guarantees a good credibility of the machine with respect to the visitors.

Within the Expo.02, the Swiss National Exhibition, the Robotics exhibition takes place in Neuchâtel, where the main thematic is nature and artifice. Robotics is intended to show the increasing proximity between man and machine. The visitors interact with up to 11 autonomous, freely navigating tour guide robots, which present the exhibit going from industrial robotics to cyborgs on a surface of $320 \mathrm{~m}^{2}$.

\section{Related Work}

The tour-guide robot task can be subdivided in two separate issues, which are navigation and interaction.

Navigation: A limited number of researchers have demon- strated autonomous navigation in exhibitions or museums [4], [11], [14], [7] and [15]. Most of these systems have still some limitations in their navigation approaches. For instance Rhino [4] and Minerva [14] have shown their strengths in museums for one week (19 kilometers) and two weeks (44 kilometers) respectively. However, their navigation has two major drawbacks: it relies on off-board resources, and due to the use of raw range data for localization and mapping it is sensible to environmental dynamics. Sage [11], Chips, Sweetlips, Joe and Adam [15], use a completely different approach for permanent installations in museums: the environment is changed by adding artificial landmarks to localize the robot. This approach performed well, as shown with a total of more than half a year of operation and 323 kilometers for Sage [11] and a total of more than 3 years and 600 kilometers for Chips, Sweetlips, Joe and Adam [15]. However their movements, but for Adam, are limited to a predefined set of unidirectional safe routes in order to simplify both localization and pathplanning. Another permanent installation which is operating since March 2000 is presented in [7]. Three self-contained mobile robots navigate in a restricted and very well structured area. Localization uses segment features and a heuristic scheme for matching and pose estimation.

Interaction: Human-centered and social interactive robotics is a comparatively young field in mobile robotic research. However, several experiences where untrained people and robots meet are available. The analysis of the first public space experience with Rhino [4] underlines the importance of improving human-robot interfaces in order to ease the acceptance of robots by the visitors. In [14] Minerva attracted visitors and gave tours in a museum. It was equipped with a face and used an emotional state machine with four states to improve interaction. The Mobot Museum Robot Series [11] and [15] focused on the interaction. Robustness and reliability were identified as an important point for the credibility of a public robot. The permanent installation at the Deutsches Museum für Kommunikation in Berlin [7], uses three robots which have the task to welcome visitors, offer them exhibition-related information and to entertain them.

The system presented here is designed to offer enhanced interactivity with complete autonomous navigation in a completely self-contained robot and without requiring changes of the environment. Furthermore it is intended to work permanently with minimal supervision. 


\section{Design}

The typical environment of an exhibit, which is highly dynamic, and the visitor experience expected with such a robot impose various constraints on the design and control. This leads to the following specification of the mobile platform:

- Highly reliable and fully autonomous navigation in unmodified environments crowded with hundreds of humans.

- Bidirectional multi-modal interaction based on speech (English, German, French and Italian), facial expressions and face tracking, icons (LED matrix), input buttons, and robot motion.

- Safety for humans, objects, and the robot itself all the time.

- Minimal human intervention and simple supervision.

The esthetic of the robot has been designed in collaboration with artists, industrial designers, and scenographers. The result of the design of both hardware and software is RoboX: a mobile robot platform ready for the real world (figure 1).

Given the above mentioned specifications, the mechanical, electronic, and software design are now presented.

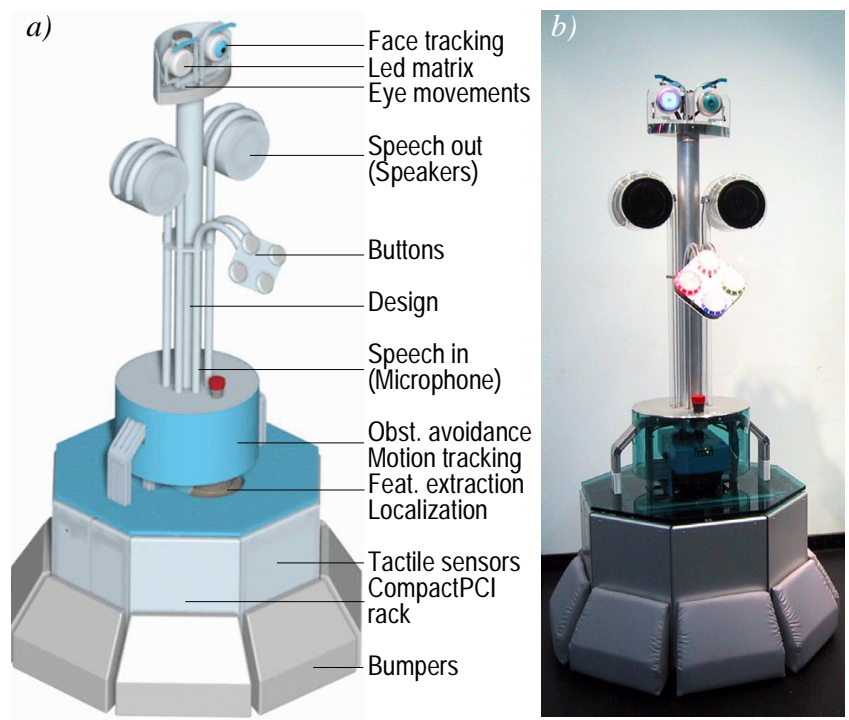

Figure 1: a) Functionality of the tour guide robot RoboX. b) An image of RoboX 9.

\subsection{Mechanical Design}

The navigation base (lower part of the robot) consists mainly in a CompactPCI rack with two control computers, two laser range sensors (SICKs LMS-200), the batteries, eight bumpers and the differential drive actuators with harmonic drive gears. The base (figure 2) has an octagonal shape with two actuated wheels on a central axis and two castor wheels. In order to guarantee good ground contact of the drive wheels, one of the castor wheels is mounted on a spring suspension. This gives an excellent manoeuvrability and stability to the $1.65 \mathrm{~m}$ high robot.

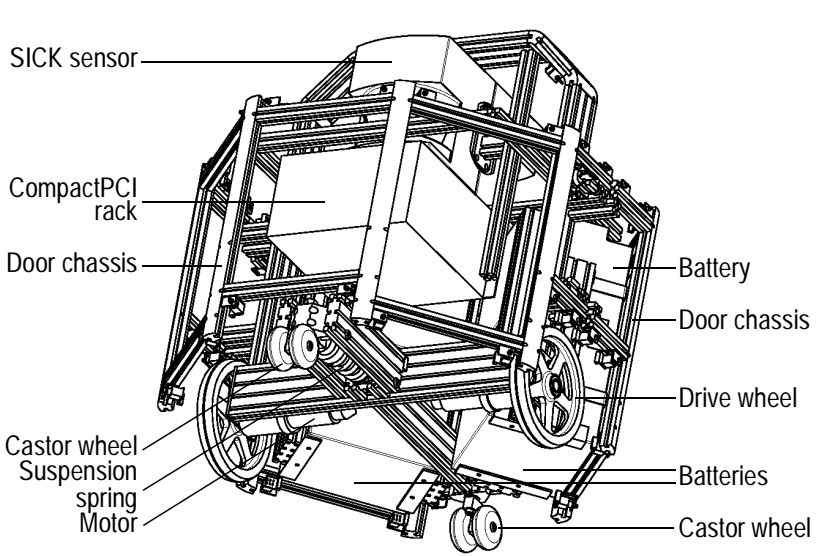

Figure 2: Mechanical design of the RoboX base.

The upper part of the robot incorporates the interaction modules. The face includes two eyes with two independently actuated pan-tilt units and two mechanically coupled eyebrows. The left eye is equipped with a color camera, which is used for face tracking. The right eye integrates a LED matrix for displaying symbols and icons. The eyebrows further underline eye expressions by means of a rotational movement. Behind the face, a gray scale camera pointing to the ceiling is mounted for localization purpose.

The main input device for establishing a bidirectional communication with the humans are four buttons which allow the visitors to reply to questions the robot asks. The robot can further be equipped with a directional microphone matrix for speech recognition even though this remains challenging in the very noisy environment of an exhibition.

\subsection{Electronic Design}

The control system (figure 3 ) has been designed very carefully by keeping in mind that the safety of the humans and the robot has to be guaranteed all the time. It is composed of a CompactPCI rack containing an Intel Pentium III card and a Motorola PowerPC 750 card. The latter is connected by the PCI backplane to an analog/digital I/O card, a Bt848-based frame grabber, an encoder IP module and a high bandwidth RS-422 IP module. Furthermore a Microchip PIC processor is used as redundant security system for the PowerPC card.

The navigation software runs on the hard real-time operating system XO/2 [3] installed on the PowerPC. This processor has direct access to the camera looking at the ceiling, the two SICK sensors, the tactile plates and the main drive motors. It communicates with the interaction PC through Ethernet via an on-board hub.

The interaction software is running under Windows 2000 on an industrial PC. This allows using commercial off-theshelf (COTS) software for speech synthesis and recognition, and makes scenario development easier. The PC has direct access to the eye camera, the eyes and eyebrows controller, the input buttons, the two loudspeakers, and the microphone.

The robot (both CPUs) is connected by a radio Ethernet to an external computer for supervision only, in order to track its status at any time on a graphical interface. 


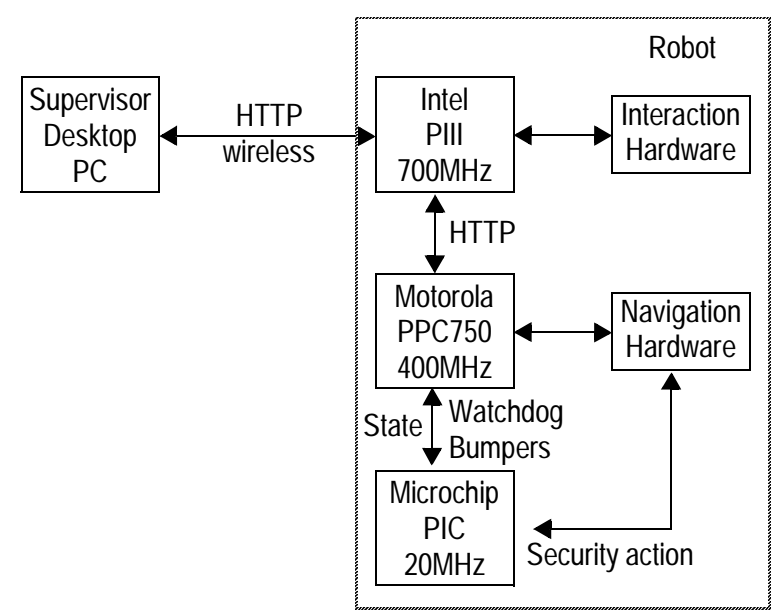

Figure 3: Simple scheme of the electrical design

\subsection{Software Design}

As explained in the section above, the robot is composed of both an Intel Pentium and a Motorola PowerPC system. The software has been designed without taking into account this fact based on the functionality which was to be developed. However, as soon as the implementation started, the objects have been assigned to one of the two distributed embedded systems. For hardware related objects (mainly sensor drivers) the choice was obvious. For the others, their relevance to safety has been evaluated: due to the hard real-time characteristics of $\mathrm{XO} / 2$, all the time-critical objects in relation with the security have been implemented on the PowerPC. Objects requiring COTS components have been implemented on the Windows machine because of their wider availability (f.e. MBrola for speech out, small FireWire camera in the eye for face tracking, etc.).

The resulting object distribution is represented in figure 4 . In the following part of this section each component of figure 4 is briefly presented starting with the interaction system followed by the navigation. A complete description of the interaction of RoboX can be found in [9]. Its navigation system is presented in [2].

\section{Interaction}

Scenario Controller: It is the central object of the interaction subsystem, which accesses all the other objects. A scenario is a sequence of tasks of all modalities (speech, face expression, motion, LED matrix, etc.). A sophisticated tourguide scenario consists of several small scenarios which are played by the scenario controller.

People detection: It permits to detect movements of objects around the robot by means of the laser scanners. By assuming a static environment, these moving objects are either humans or other robots. The moving objects are then tracked by means of Kalman Filters.

Speech Out: By using software permitting either text-tophonemes-to-speech or directly text-to-speech, this object permits the robot to talk in four languages (English, German, French, and Italian). Furthermore, files of format .wav and .mp3 can be played.
Buttons Controller: This controls the main input device for the interaction between the robot and the humans. Four capacitive buttons with different colored lights are used in combination with questions from the speech out to close the interaction loop.

LED Matrix: The LED matrix is in the right eye. Its controller permits to show icons and animations.

Eyes Controller: The eyes can be moved independently. The controller has a set of predefined expressions, which can be directly played.

Face Tracking: The color camera in the left eye is used to track skin colored regions. The approach is based on [8]. In combination with the eyes controller, this permits to track a face on the image and with the movement of the eyes.

\section{Navigation}

Odometry Driver: Calculates the position and uncertainty of the robot based on the wheel rotations.

Speed Controller: Regulates the speed defined by the obstacle avoidance with a PID controller accessing the encoders and updates the odometry.

Localization: Uses a new approach [1] based on an Extended Kalman Filter [5] to correct the odometry with exteroceptive sensors (laser scanners, CCD camera).

Obstacle Avoidance: Calculates a collision free path by initializing the path with a NFI function [10] and using the Elastic Band approach [12] to dynamically adapt it. Furthermore it guarantees that the robot can stop before collision at any time with the Dynamic Window approach [6].

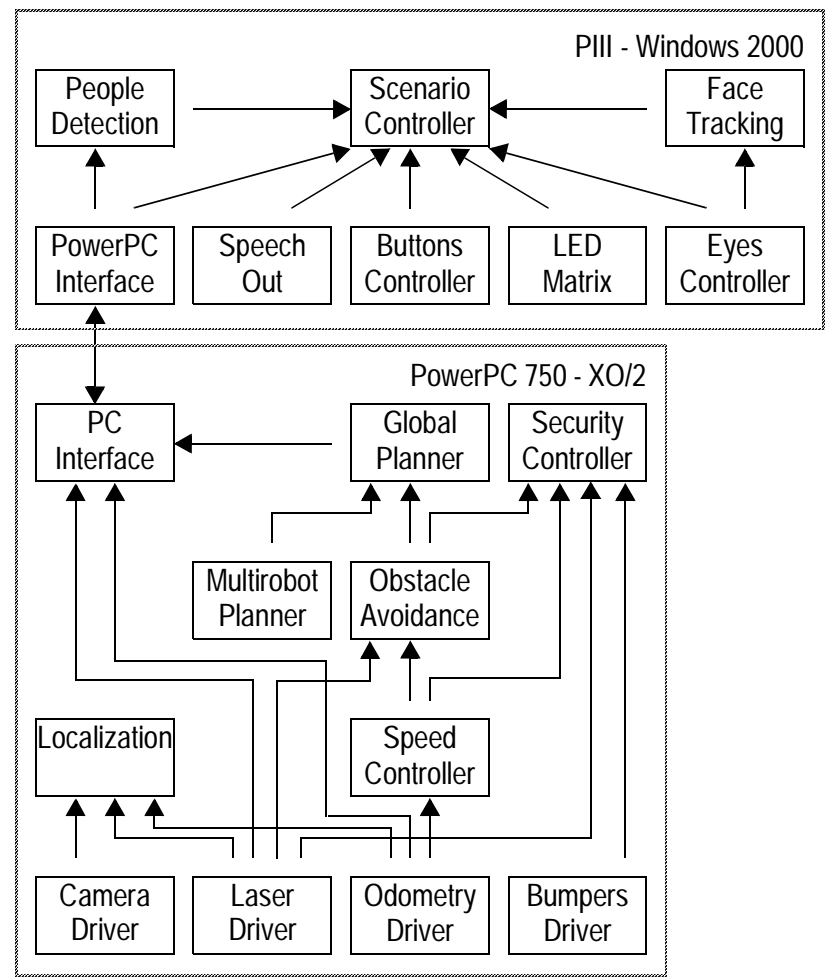

Figure 4: Object distribution of the software on the distributed embedded system. 
Multirobot Planner: Synchronizes the movement of the robots to avoid having many robots going to the same place.

Global Planner: Plans the navigation of the robot on the a priori map level, by defining via points which permit to reach the goal point within the graph representing the map.

Security Controller: Guarantees that the robot cannot become dangerous even in case of failure, by supervising the safety-critical software and sensors. Due to the importance of this issue for a robot sharing the environment with humans, the next section presents the security system in details.

\section{Security}

In this section the involvement of the security issue in the design of the whole system is pointed out in more details.

All the software which relates to the movement of the robot is defined as safety critical. In order to guarantee the security of both the users and the robot itself, safety is on three levels: the operating system, the software implementation and the redundancy of the hardware.

\subsection{Operating System}

All the navigation software is implemented on the Power$\mathrm{PC}$ which is operated by $\mathrm{XO} / 2$, a deadline driven hard realtime operating system [3]. Due to its characteristics XO/2 helps for all the components [13] of safety, which are:

- Safety: nothing bad happens.

- Progress: the right thing do (eventually) happen.

- Security: things happens under proper supervision.

Static safety is guaranteed by the strong-typing characteristic of Oberon-2, the language used under XO/2. Many errors are already found at compile-time instead of run-time. Furthermore, index-checks, dynamic type systems and especially the real-time compatible garbage collector guarantee dynamic safety by forbidding almost any memory-management related errors.

The deadline driven scheduler is in charge of progress: it guarantees that each task is executed within the predefined deadline. Of course this is possible only if the constellation of the tasks running on the PowerPC requires less than $100 \%$ of the CPU. For this, the duration of each tasks has to be known. Admission tests are performed at each installation of a new real-time task to guarantee their feasibility. As soon as the progress of all real-time tasks is guaranteed, the CPU is scheduled between the non-real-time tasks depending on their priorities.

Each error causes a system trap which is under complete control of the operating system. The system knows exactly where the error took place, who called this part of the code up to the task currently running (stack trace). This is very helpful for debugging, but it is even more important for security because for each task an exception handler can be defined. The actions which have to take place in such a case can therefore be properly defined.

\subsection{Software Security}

Tasks whose failure could cause injuries to people or damage objects required special attention during design. Soft- ware watchdogs are therefore implemented for the speed controller, the obstacle avoidance, the laser driver and the bumpers driver (figure 4). Failure of one of these tasks is detected by the security controller which then either restarts the failed task or stops the robot, turns on the alarm blinker and sends an e-mail to the maintenance. This permit to centralize the control of the security and to refer to a single object if a problem occurs. Furthermore, the security controller generates a watchdog signal on a digital output permitting to know if both the operating system and the security controller are still running.

\subsection{Hardware Redundancy}

The above mentioned software permits to have a consistent control system running on the PowerPC. However, this isn't enough to guarantee the security of the robot and its surrounding. Even in case of failure of the electronics or problems on the operating system of the PowerPC, the robot must remain un-dangerous. For this, the robot has a third processor: a Microchip PIC (figure 3). The software running on it checks the watchdog generated by the security controller, awaits acknowledgements from the security for each bumper contact and controls that the pre-defined maximal speed is never exceeded. If one of these conditions is not respected the redundant security software running on the PIC safely stops the robot (it shortcuts the phases of the motors) and puts it in emergency mode (acoustic alarm).

\section{Experiments}

The whole operational period, 159 days from May 14 to September 17, is available for statistics. Each day from six to eleven freely navigating tour-guide robots have given tours from 9:30am to 8:00pm until August, then from 9:00am to 8:00pm in September and from 9:00am to 9:00pm in October on the surface of the exhibit which is approximately $320 \mathrm{~m}^{2}$.

\subsection{Definitions}

Failure: A failure is any kind of problem which requires human intervention. The only exceptions are the emergency button, which can be pressed and released also by visitors, and the situations where the robot remains blocked because it is to near too an object. In the latter case, the staff can displace the robot by a switch which disconnects the motors from the amplifiers and allows to move the 115 kilograms robot easily.

Uncritical: Uncritical failures are those which do not stop the task of the robot. For example, a failure consisting in a robot which stops sending an image to the supervisor is not critical for the tour the robot is giving to the visitors.

Critical: Critical failures stop the robot until human intervention is performed. An example is the failure of the scenario controller or of the obstacle avoidance.

Reboot: Critical failures requiring a reboot of either the Pentium or the PowerPC are treated separately because they require more time before the robot is again operational. 


\subsection{Results}

During the 159 days of operation the robots served more than 680 '000 visitors for a total of 13'313 hours of operational time. In order to perform their job, they travelled 3'316 kilometers for a total moving time of more than 9'415 hours meaning that the mean displacement speed is 0.098 meters per second. As it can be seen in table 1, the uncritical failures represent only a small portion of the total amount of failures $(6.7 \%)$. Furthermore they do not disturb the operation of the robot. They are therefore not treated in the following analysis which will focus on the critical and reboot failures of the whole robot first and then of the PowerPC.

\begin{tabular}{|c|c|}
\hline Run time & $13^{\prime} 313 \mathrm{~h}$ \\
\hline Movement time & 9’415 h \\
\hline Travelled distance & 3’316 km \\
\hline Speed (average / max) & $0.098 / 0.6 \mathrm{~m} / \mathrm{s}$ \\
\hline Failures (total / critical / uncritical) & $4{ }^{\prime} 378 / 44^{\prime} 086 / 292$ \\
\hline Critical failures (PC / PPC / HW) & $3{ }^{\prime} 216 / 694 / 176$ \\
\hline Visitors & $686 ' 405$ \\
\hline
\end{tabular}

Table 1: Five months of operation. More than 13'000 hours of work, where the RoboXes have travelled 3'300 kilometers and served more than 680'000 visitors.

As it can be seen in figure 5, the beginning of the exposition in the middle of May showed that some work was still to be done. The software running on the PC was very unstable due especially to errors in treating the list of the tasks running in the scenario controller.

The mean time between failure (MTBF) of the whole robot (PC, PowerPC and hardware) during the first three weeks was 1.41 hours. This has improved to 4.61 hours from week four to the end, which means that during one day with 10 robots, the staff had to perform a mean of 25 interventions. The type of interventions goes from the simple double-click to restart an application (typical intervention on the PC) to the change of a motor amplifier (very rare, it happened five times, two of them due to a motor defect). After the first three weeks, the MTBF already doubled.

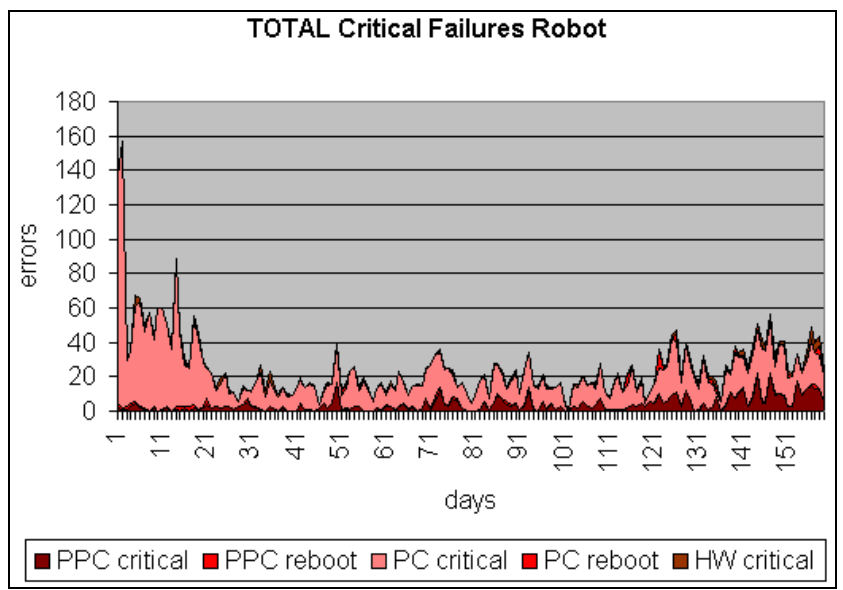

Figure 5: Due to many delays in the development, the software was still in the test phase at the beginning of the exposition. The first three weeks represent a huge improvement in the stability of the software, especially on the PC side.

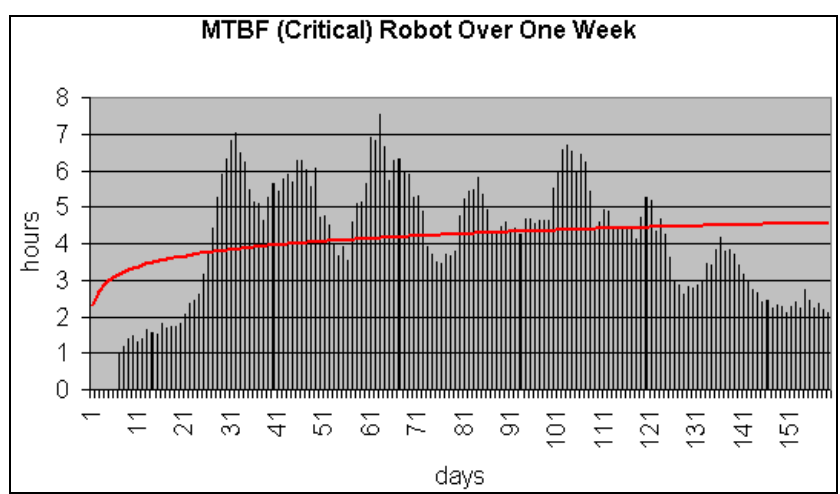

Figure 6: The mean time between critical failure of any kind (PC, PowerPC, hardware). The improvement has been constant exponential during the first four weeks, where the most important errors have been found.

In figure 7 all the critical failures coming from the navigation software (PowerPC) are shown. During the first three weeks, errors in the safety-critical tasks were treated by the security controller, but could sometimes require a reboot in order to restart the trapped task. This has been partly corrected allowing for much faster intervention in case of failure. Critical failures in figure 7 also contain errors which have not directly to do with the software: situations where the robot went lost. The main reason for lost-situations are visitors or untrained staff members who handle the robots without using the switch to disconnect the motors from the amplifiers during a manual intervention. This causes unmodeled odometry errors of such an extent that the robot went lost (robot kidnapping). Note that this type represents $73 \%$ of the critical failures of the PowerPC (504 failures) and that they are not software failures, but situations which the localization system cannot handle since underlying assumptions are violated (more details in [2]).

The MTBF for the PowerPC (figure 8 (a)) was between 20 and 80 hours already at the beginning of the exposition. By taking into account only the software errors (figure 8 (b)), the MTBF over the whole period is 70.1 hours.

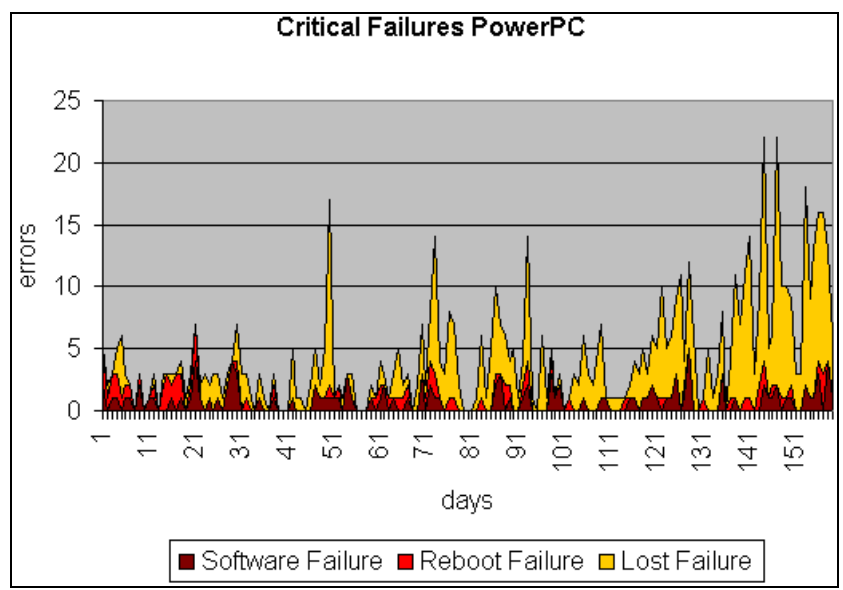

Figure 7: The critical failures of the PowerPC (navigation system). Some of the critical errors require the reboot of the PowerPC. Lost failures are not software errors (they are not "bugs"). 


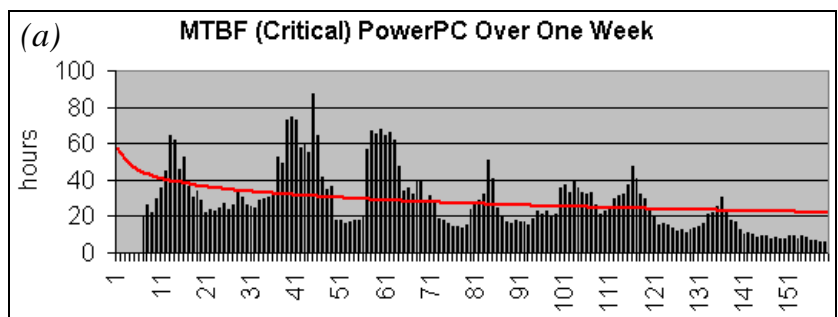

(b) MTBF (Critical - Lost Situations) PowerPC Over One Week

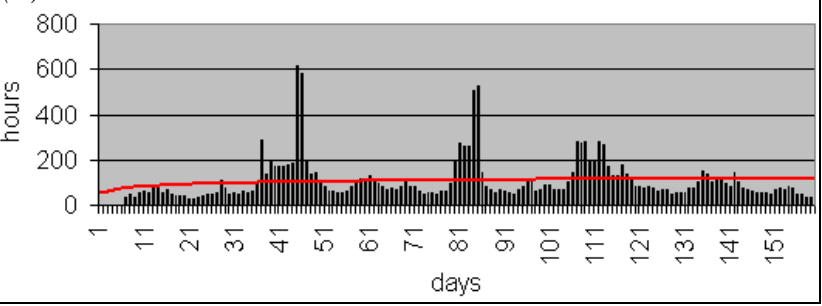

Figure 8: The MTBF (critical) with (a) and without (b) lost situations. By not taking into account the lost situations (b) the MTBF is very high (mean 70.1 hours).

Hardware failures (figure 9) are due to some uncritical design errors at the beginning (robot doors), some motor-amplifier problems and to the high temperature between day 33 and day 40 causing some component failures.

\subsection{Lessons to be Learned}

The characteristics of this project give an extraordinary chance of learning by experience. Thousands of hours of operation permit to improve the software and hardware to a level which is simply not achievable in smaller projects. This was shown during the exploitation, where some errors were found after few days of operation while others appeared for the first time after one or two months. The best example are the failures of the laser scanners on week 5 due to the temperature in the exhibit. This failure wasn't taken into account by the security causing the obstacle avoidance to permanently receive the last available scan and the robot to collide with the next encountered object. This problem is since then under supervision of the Security Controller (figure 4).

Another interesting point is the difference in the software reliability implemented under PC and PowerPC. The better result of the PowerPC is due to the real-time $\mathrm{XO} / 2$ operating system which has been developed for embedded systems focusing on robustness and safety [3], and also to the longer experience in navigation at the Autonomous Systems Lab in contrast to the new interaction software which has been developed only for this application starting in late year 2000 .

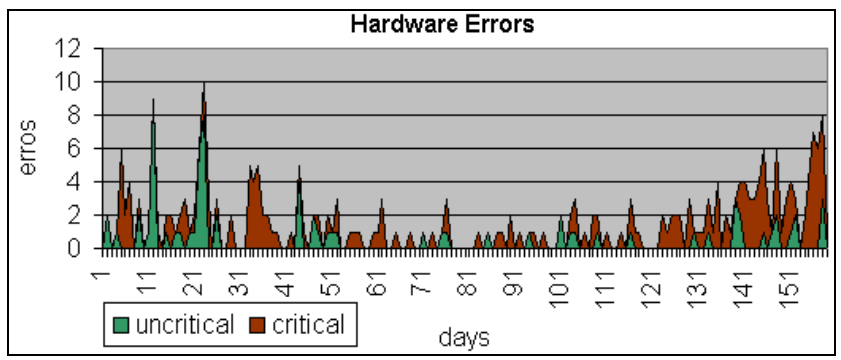

Figure 9: Hardware problems also cause critical failures.

\section{Conclusion}

This project represents a milestone in the field of mobile robotics: for the first time interactive mobile robots are produced (11 robots) and used for a long time (five months) as real products instead of prototypes as in former projects. The paper presents their characteristics first, then goes into details about the mechanical, electrical and software design. The security issue is faced seriously for ensuring security of the humans and the robot itself all the time. In the experiments section the results of the whole project (159 days of operation) of the Robotics exposition are presented and analyzed focusing on the amount and type of robot failures.

\section{References}

[1] Arras, K. O., J. A. Castellanos, and R. Siegwart (2002). Feature-Based Multi-Hypothesis Localization and Tracking for Mobile Robots Using Geometric Constraints. IEEE International Conference on Robotics and Automation, Washington DC, USA.

[2] Arras, K. O., R. Philippsen, N. Tomatis, M. De Battista, M. Schilt, and R. Siegwart (2002). A Navigation Framework for Multiple Mobile Robots and its Application at the Expo.02 Exhibition. IEEE International Conference on Robotics and Automation, Taipei, Taiwan.

[3] Brega, R., N. Tomatis, K. Arras, and R. Siegwart (2000). The Need for Autonomy and Real-Time in Mobile Robotics: A Case Study of XO/2 and Pygmalion. IEEE/RSJ International Conference on Intelligent Robots and Systems, Takamatsu, Japan.

[4] Burgard, W., A. B. Cremers, et al. (1999). "Experiences with a Interactive Museum Tour-Guide Robot." Artificial Intelligence 00(1999): 1-53.

[5] Crowley, J. L. (1989). World Modeling and Position Estimation for a Mobile Robot Using Ultrasonic Ranging. IEEE International Conference on Robotics and Automation, Scottsdale, AZ.

[6] Fox, D., W. Burgard, et al. (1997). "The Dynamic Window Approach to Collision Avoidance." IEEE Robotics \& Automation Magazine: 23-33.

[7] Graf, B., R. D. Schraft, et al. (2000). A Mobile Robot Platform for Assistance and Entertaiment. International Symposium on Robotics, Montreal, Canada.

[8] Hilti, A., I. Nourbakhsh, B. Jensen, and R. Siegwart (2001). Narrative-level Visual Interpretation of Human Motion for Human-robot Interaction. IEEE/RSJ International Conference on Intelligent Robots and Systems, Maui, Hawaii.

[9] Jensen, B., G. Froidevaux, X. Greppin, A. Lorotte, L. Mayor, M. Meisser, G. Ramel and R. Siegwart (2002). The interactive autonomous mobile system RoboX. IEEE/RSJ International Conference on Intelligent Robots and Systems, Lausanne, Switzerland.

[10] Latombe, J.-C. (1991). Robot motion planning. Dordrecht, Netherlands, Kluwer Academic Publishers.

[11] Nourbakhsh, I., J. Bodenage, et al. (1999). "An Effective Mobile Robot Educator with a Full-Time Job." Artificial Intelligence 114(1-2): 95-124.

[12] Quinlan, S. and O. Khatib (1993). Elastic bands: connecting path planning and control. IEEE International Conference on Robotics and Automation.

[13] Szyperski, C. and J. Gough (1995). The role of programming languages in the life-cycle of safe systems. Second International Conference on Safety Through Quality (STQ'95), Kennedy Space Center, Cape Canaveral, Florida, USA.

[14] Thrun, S., M. Beetz, et al. (2000). "Probabilistic Algorithms and the Interactive Museum Tour-Guide Robot Minerva." International Journal of Robotics Research 19(11): 972-99.

[15] Willeke, T., C. Kunz, et al. (2001). The History of the Mobot Museum Robot Series: An Evolutionary Study. Florida Artificial Intelligence Research Society (FLAIRS), Florida. 